\title{
Austrian Society of Gastroenterology and Hepatology (ÖGGH) - Guidelines on sedation and monitoring during gastrointestinal endoscopy
}

Authors

Institution

\section{F. Schreiber, The Working Group on Endoscopy, Austrian Society of Gastroenterology and Hepatology (ÖGGH)}

Division of Gastroenterology and Hepatology, Department of Internal Medicine, Medical University of Graz

\section{Bibliography}

Dol $10.1055 / \mathrm{s}-2007-966254$

Endoscopy 2007; 39;

259-262 @ Georg Thieme

Verlag KG Stuttgart · New York ISSN 0013-726X

\section{Corresponding author \\ F. Schreiber, MD}

Division of Gastroenterology and Hepatology

Department of Internal Medicine Medical University of Graz Auenbruggerplatz 15 8036 Graz

Austria

Fax: +43-316-385-2648

florian.schreiber@ meduni-graz.at

\section{Introduction \\ $\nabla$}

The increasing invasiveness of endoscopic examinations and interventions means that adequate sedation and appropriate patient monitoring are required. Overall, endoscopic interventions in the gastrointestinal tract are low risk, provided that they are performed in sufficient numbers by experienced personnel [1]. The risk of the examination or intervention depends primarily on the invasiveness and technical complexity of the procedure, but also on the patient's individual risk profile and the specific side-effect profile of the sedatives or anesthetics used. The overall rate of serious complications in gastroenterological examinations and interventions is between 1 in 1000 and 1 in 7500 [2].

The quality of procedures and, in turn, patient safety depend greatly on precise and careful risk estimation before, during, and after the procedure. Information provided to patients on the planned procedure should not only be timely and cover the invasiveness of the procedure - it should include information not only on the risks of the procedure itself but also on the specific risks of sedation.

\section{Risk factors \\ $\nabla$}

For the evaluation of the entire risk of a procedure, a clear distinction must be made between general risk (patient-specific factors), risks inherent in the procedure itself (for example pancreatitis after endoscopic retrograde cholangiopancreatography [ERCP], perforation and/or bleeding after papillotomy, and bleeding after endoscopic muco-resection), and the risks associated with sedation.

The general risk for upper gastrointestinal endoscopy depends mainly on the presence of cardiorespiratory disease and on the general health of the patient $[3,4]$. In this context, the complication rate increases very considerably with the length of the procedure.

Before every procedure, patient-specific risk factors must be evaluated. This is best done on the basis of the classification of the American Society of Anesthesiologists (ASA) ( $\square$ Table 1 ). Before every procedure, in the context of obtaining the patient's informed consent, the patient's history and medical reports must be obtained. The spectrum of clinical and preparatory studies to be ordered increases with increasing risk (see - Table 2).

\section{Sedation \\ $\nabla$}

Different degrees of sedation have been defined: "sedation" is understood to mean a clouding of consciousness; "deep sedation" is loss of consciousness with retention of spontaneous respiration and protective reflexes; and "general anesthesia" is defined as loss of consciousness, spontaneous respiration, and protective reflexes, attributable to the effects of substances acting on the central nervous system.

The increasing technical complexity of endoscopic procedures increasingly commonly requires deep sedation of the patient as well as adequate analgesia. Even for purely diagnostic endoscopy, sedation can be advantageous, not only for the patient but also in terms of achievement of higher-quality procedures. The following list summarizes the endoscopic procedures which most often require the use of sedation or deep sedation:

1. Interventional endoscopy in the upper gastrointestinal tract (hemostasis; treatment of varices with ligation, sclerotherapy, or tissueadhesive therapy; dilation procedures; implantation of prostheses; endoscopic mucosal resection; and disobliteration procedures); 
Table 1 The American Society of Anesthesiologists (ASA) classification of anesthetic risk according to general health status

\begin{tabular}{|ll|}
\hline ASA class & Clinical profile \\
\hline I & Healthy patient \\
\hline II & Mild illness without physical limitations \\
\hline III & Serious illness with physical limitations \\
\hline IV & Serious illness with threat to life \\
\hline V & Death expected within 24 hours \\
\hline
\end{tabular}

2. diagnostic and interventional endosonography;

3. percutaneous endoscopic gastrotomy;

4. colonoscopy with difficult and/or multiple polypectomy or with endoscopic mucosal resection;

5. ERCP with interventions involving the bile ducts (endoscopic papillotomy, mechanical lithotripsy, stone removal, implantation of prostheses) and the pancreatic ducts (selective sphincterotomy, stone removal, dilation, implantation of prostheses).

With purely diagnostic gastroscopy, the use of sedatives involves a slightly higher rate of complications [5,6], but patient acceptance is significantly higher than without sedation.

Benzodiazepines are the most commonly used sedatives; opiates have been used much less frequently in recent years due to the high rate of respiratory complications.

Midozalam is a sedative that was specially developed for short procedures [7]; besides sedation, it provides antegrade amnesia, a desirable effect that means that the unpleasant sensations perceived by the patient during endoscopy tend to recede. Its use does, however, require that special attention is paid to the outpatients who receive it, especially with regard to the provision of patient information and patient handling after endoscopy (see below).

The increasing complexity of endoscopic procedures in recent years has necessitated the use of centrally acting anesthetics. Propofol is a particularly popular choice for induction and maintenance of deep sedation. It is a highly lipophilic anesthetic with fast distribution (2-4 minutes) and fast elimination (half-life 30-60 minutes). The therapeutic spectrum of propofol, however, is much narrower than that of midazolam, so that careful monitoring to differentiate between sedation, deep sedation, and narcosis is much more demanding as far as personnel and equipment are concerned.

\section{The safe use of sedatives and anesthetics}

The patient's safety must take the highest priority in all considerations surrounding the use of a medication.

A meta-analysis of randomized studies published to date comparing propofol and conventional sedatives did not show a higher complication rate for propofol, but it did reveal that recovery after propofol was significantly faster and also a trend toward a lower incidence of hypoxia and hypotension (though this finding was not statistically significant) [8]. This leads to the conclusion that propofol is at least as safe as the generally accepted conventional sedation with benzodiazepine derivatives or midazolam.

Although anesthesiologists the world over have repeatedly stressed that only they are in a position to use an anesthetic such as propofol safely, there are data indicating that this drug can be used safely by nonanesthesiologists $[9,10]$. In the earlier of these two studies, propofol was administered over a period of 5 years for 28472 endoscopic procedures, either by general medical staff or by anesthesiologists [9]: there were 185 complications related to sedation $(0.64 \%)$ but no deaths. There were no specific differences between the two types of physicians with regard to the figures for complications. In the 2003 study [10], the sedation-related complication rate did not increase when propofol was administered to 819 patients (ASA I-IV) by gastroenterologists.

There are also well-documented data showing that this regime is safe when propofol is administered by specially trained nursing personnel $[11-13,18]$. No disadvantageous effects were seen when propofol was administered by specially trained nursing personnel to 9152 patients undergoing outpatient endoscopy. A further prospective study covering 27500 documented cases did not show any negative effect on the complication rate when propofol was given by nurses. In one controlled study, this was shown to be true even for high-risk patients (ASA III/IV) [14]. These data are taken into consideration in the revised "Practice guidelines for sedation and analgesia by non-anesthesiologists", published by the American Society of Anesthesiologists in 2002 [17]. The relevant recommendation reads, "An additional person, specially entrusted with this task, must be present, who is qualified to safely administer and monitor the sedation, and to take appropriate emergency measures..."

One prerequisite for the safe use of sedatives and anesthetics is safe venous access. Apart from the administration of the sedative agent, another important safety factor when considering the use of propofol is the provision of adequate monitoring during deep sedation and after completion of the endoscopy or endoscopic intervention.

It is obvious that the endoscopist cannot be expected to simultaneously perform the endoscopic procedure, which may be very complex; administer an anesthetic with a narrow therapeutic spectrum; and monitor the patient in a dimly lit endoscopy unit. There must be an additional person present in the endoscopy suite whose sole responsibility is to administer the sedative or anesthetic and to monitor the patient during the endoscopy. According to published data, this person can be an anesthesiologist, a specially trained physician, or a specially trained member of the nursing staff. The specially trained nurses must be familiar with the agent administered, be able to maintain respiration when complications occur or during the transition from deep sedation to general anesthesia, and be able to handle cardiovascular side effects or complications caused by the agent administered.

In all cases, there should be complete, clear, and understandable documentation of all measures taken when a sedative or anesthetic is administered.

\section{Monitoring}

With deeper sedation, it is absolutely essential to provide for suitable monitoring during endoscopy or endoscopic interventions. Monitoring of the patient is primarily the task of the designated staff member (medical or nursing personnel); technical equipment is only supplementary.

Routine endoscopy with conventional sedation calls for continuous, noninvasive oxygen monitoring (pulse oximetry), though there have been no controlled studies proving that cardiorespiratory complications are reduced when this measure is em- 


\section{ASA class III/IV \\ Decompensated heart failure, NYHA class III/IV \\ Coronary heart disease \\ Valve disease/replacement \\ Hepatic and renal failure \\ Pulmonary disease \\ Clotting disorders \\ ASA, American Society of Anesthesiologists; NYHA, New \\ York Heart Association.}

Table 3 Patients in whom the physician might anticipate a "difficult" endotracheal intubation (according to the American Society of Anesthesiologists)

Patients with previous complications related to sedation or anesthesia

Patients with stridor, known sleep apnea, known tracheomalacia, or

tracheal stenosis

Patients with congenital deformities of the nasopharynx (e.g. trisomy

21, Pierre Robin sequence)

Patients with relevant dental, oral, or jaw malformations

Patients with congenital or acquired conditions of the cervical spine

ployed [15]. This would be hard to prove in view of the low rate of complications attributable to endoscopy and sedation and consequently the very large number of patients that would be required in any study.

Routine administration of oxygen is also controversial. It has been shown that the average oxygen saturation decreases, but not that severe hypoxia can be effectively prevented [16]. The benefit of continuous, noninvasive blood-pressure monitoring under conventional sedation has also not been proved.

When propofol or deep sedation is used, equipment must be available for mask respiration and endotracheal intubation; the medications for resuscitation should be at hand and there should be oxygen and vacuum connections. With this regime, apart from the continuous administration of oxygen, there is no question of the value of continuous, noninvasive blood-pressure measurement, because hypotension as a specific side effect of propofol that can and should be recognized promptly. Electrocardiographic monitoring should also be available.

When patients with known risk factors undergo endoscopy or endoscopic interventions under deep sedation who might require endotracheal intubation (see $\bullet$ Table 3 ), the presence of an anesthesiologist might be desirable, depending on the intubation experience of the endoscopy team.

In all cases there should be complete, clear, and understandable documentation of all parameters recorded during the entire endoscopic procedure.

\section{Postendoscopic surveillance \\ $\nabla$}

The sedative effect of the agents used persists far beyond the end of the endoscopy or endoscopic intervention. Surveillance will depend on which agents have been used and the depth of sedation, and should be continued until the patient has completely recovered consciousness. There should be a suitably equipped and staffed recovery room. Pulse oximetry should be available, as should electrocardiography and noninvasive blood-pressure monitoring (for high-risk patients) after administration of propofol.

Outpatients should only leave the recovery room when they have fully regained consciousness. The patient will have been in- formed in advance that for the 24 hours following sedation he or she should avoid business transactions, operating motor vehicles, and performing difficult or dangerous tasks; nonetheless, these instructions should be repeated before the patient is discharged.

Here as well there should be clear and careful documentation of all parameters recorded and all measures taken.

\section{Summary}

1. Patient safety has the highest priority in the performance of endoscopy or endoscopic interventions and in the administration of accompanying measures such as sedation. This applies above all to the allocation of time, space, personnel, and equipment in the endoscopy suite.

2. Every procedure must be preceded by an individual risk classification assessment. The result must be recorded in writing (in the context of the information given to the patient).

3. Good venous access is a prerequisite.

4. The administration of sedatives or anesthetics must, above all, take into consideration the depth of sedation. This in turn determines the extent of monitoring. The medication and comedications used (including trade names and dosage) must be documented, either in the endoscopy report or in a separate record.

5. There must always be an individual present who is responsible for the administration of sedatives or anesthetics. Depending on the degree of sedation on the one hand and on the presence of risk factors that could lead to a requirement for intubation on the other, this individual can be a specially trained assistant or nurse, a member of the general medical staff, or an anesthesiologist.

6. This individual is responsible for monitoring during the endoscopic procedure. The patient's recovery must be monitored in a specially equipped unit and be briefly documented by the responsible staff there.

7. Special attention must to given to precise and detailed documentation of all steps of the process described (risk stratification, patient information, medication, procedural records).

\section{Competing interests: None}

\section{References}

1 Sieg A, Hachmöller-Eisenbach U, Eisenbach Tet al. Prospective evaluation of complications in outpatient GI endoscopy: a survey among German gastroenterologists. Gastrointest Endosc 2001; 53: 620-627

2 Daneshmend TK, Bell GD, Logan RF. Sedation for upper GI endoscopy: results of a nationwide survey. Gut $1991 ; 32$ : $12-15$

3 Arrowsmith JB, Gerstmann BB, Fleischer DE, Benjamin SB. Results from the American Society for Gastrointestinal Endoscopy/US Food and Drug Administration collaborative study on complication rates and drug use during gastrointestinal endoscopy. Gastrointest Endosc 1991; 37: 421 - 427

4 Dinges $Y$, Hahn M, Jung $M$. Troponin I zur Erfassung subklinischer kardialer Ischämien bei der ERCP. Z Gastroenterol 2000; 38: 763

5 Fröhlich F, Schwizer W, Thorens J et al. Conscious sedation for gastroscopy: patient tolerance and cardiorespiratory parameters. Gastroenterology 1995; 108: $697-704$

6 Gatto G, Peri V, Amuso M, Traina M. Sedation is not needed for upper gastrointestinal endoscopy. Endoscopy 1996; 28 (Suppl): 41

7 Lee MG, Hanna W, Harding $H$. Sedation for upper gastrointestinal endoscopy: a comparative study of midazolam and diazepam. Gastrointest Endosc 1989; 35: $82-84$ 
8 Abraham NS, Raman M, Thompson Ket al. Treatment efficacy and safety related to the use of propofol vs. conventional sedation: a meta-analysis of published controlled trials. GI Endos 2004; 59: AB128

9 Clarke AC, Chiragakis L, Hillman LC, Kaya GL. Sedation for endoscopy: the safe use of propofol by general practitioner sedationists. Med J Aust 2002; 176: 159-162

10 Cohen L, Dubovsky A, Aisenberg J, Miller K. Propofol for endoscopic sedation: a protocol for safe and effective administration by the gastroenterologist. Gastrointest Endosc 2003; 58: 725 - 732

11 Rex KR, Overley Ch, Kinser K et al. Safety of propofol administered by registered nurses with gastroenterologist supervision in 2000 endoscopic cases. Am J Gastroenterol 2002; 97: 1159-1164

12 Walker JA, McIntyre RD, Schleinitz PF et al. Nurse-administered propofol sedation without anesthesia specialists in 9152 endoscopic cases in an ambulant surgery center. Am J Gastroenterol 2003; 98: 1744 1750

13 Heuss LT, Schnieper P, Drewe J et al. Risk stratification and safe administration of propofol by registered nurses supervised by the gastroen- terologist: a prospective observational study of more than 2000 cases. Gastrointest Endosc 2003; 57: 664-671

14 Heuss LT, Schnieper P, Drewe J et al. Safety of propofol for conscious sedation during endoscopic procedures in high-risk patients: a prospective, controlled study. Am J Gastroenterol 2003; 98: 1751 - 1757

15 Bell GD, Jones JG. Routine use of pulse oximetry and supplemental oxygen during endoscopic procedures under conscious sedation: British beef or common sense? Endoscopy 1996; 28: 718-721

16 Holm C, Rosenberg J. Pulse oximetry and supplemental oxygen during gastrointestinal endoscopy: a critical review. Endoscopy 1996; 28: $703-711$

17 American Society of Anesthesiologists. Practice guidelines for sedation and analgesia by non-anesthesiologists. Anesthesiology 2002; 96: $1004-1017$

18 Tohda G, Higashi S, Wakahara S et al. Propofol sedation during endoscopic procedures: safe and effective administration by registered nurses supervised by endoscopists. Endoscopy 2006; 38: 360-367 